\title{
Rede Amamenta Brasil e Estratégia Amamenta e Alimenta Brasil: impacto nos índices de aleitamento materno
}

\author{
Brazilian Breastfeeding Network and Brazilian Breastfeeding and Feeding Strategy: the impact on \\ breastfeeding indicators
}

Red Amamanta Brasil y Estrategia Alimentaria Brasileña de Lactancia Materna: impacto en las

tasas de lactancia

Recebido: 31/07/2021 | Revisado: 07/08/2021 | Aceito: 08/08/2021 | Publicado: 12/08/2021

Priscila Yoshida Machado ORCID: https://orcid.org/0000-0001-8902-9173 Minas Cardio Instituto de Intervenção Cardiovascular, Brasil

E-mail: pri.yoshida@hotmail.com

Nayara Girardi Baraldi

ORCID: https://orcid.org/0000-0003-0124-8174

Fundação Hermínio Ometto, Brasil E-mail: consultoriasermae@gmail.com

Cristiane Aparecida Silveira-Monteiro

ORCID: https://orcid.org/0000-0002-8427-7220

Universidade Federal de Alfenas, Brasil

E-mail: cristiane.monteiro@unifal-mg.edu.br

Nathália Gianini Nery

ORCID: https://orcid.org/0000-0002-6148-0613

Prefeitura Municipal de Alfenas, Brasil

E-mail: nathaliagianininery@gmail.com

Christianne Alves Pereira Calheiros

ORCID: https://orcid.org/0000-0002-7469-6034

Universidade Federal de Alfenas, Brasil

E-mail: christianne.calheiros@unifal-mg.edu.br

Patrícia Scotini Freitas

ORCID: https://orcid.org/0000-0002-8270-8955

Universidade Federal de Alfenas, Brasil

E-mail: patricia.freitas@unifal-mg.edu.br

\begin{abstract}
Resumo
Objetivo: Refletir sobre o impacto da Rede Amamenta Brasil e da Estratégia Amamenta e Alimenta Brasil sobre os índices de aleitamento materno no país. Método: trata-se de uma revisão narrativa da literatura desenvolvida por meio da discussão da literatura sobre a temática objetivo dessa reflexão. Resultados: foram encontrados oito estudos que respondiam a questão norteadora do estudo, sendo seis que avaliavam impacto da Rede Amamenta Brasil e dois da Estratégia Amamenta e Alimenta Brasil nos índices de aleitamento materno. Do total, seis eram artigos, um relatório de pesquisa e uma dissertação. Conclusão: os achados foram aparentemente contraditórios: enquanto algumas pesquisas demostraram melhora dos índices de aleitamento materno, outras não encontraram aumento da prevalência em unidades que aderiram a Rede Amamenta Brasil, hoje denominada Estratégia Amamenta e Alimenta Brasil.
\end{abstract}

Palavras-chave: Aleitamento materno; Políticas de saúde; Atenção primária à saúde.

\begin{abstract}
Objective: To reflect on the impact of Brazilian Breastfeeding Network and the e Brazilian Breastfeeding and Feeding Strategy on breastfeeding rates in Brazil. Method: this is a narrative review of the literature developed through the discussion of literature on the objective of this reflection. Results: eight studies were found that answered the study's guiding question, six of which assessed the impact of Brazilian Breastfeeding Network and two of the Brazilian Breastfeeding and Feeding Strategy on breastfeeding rates. Of the total, six were articles, a research report and a dissertation. Conclusion: the findings were apparently contradictory: while some studies showed an improvement in breastfeeding rates, others did not find an increase in the prevalence in units that joined the Brazilian Breastfeeding Network, now called Brazilian Breastfeeding and Feeding Strategy.
\end{abstract}

Keywords: Breastfeeding; Health policies; Primary health care. 


\section{Resumen}

Objetivo: reflexionar sobre el impacto de la Red Amamanta Brasil y Estrategia Alimentaria Brasileña de Lactancia Materna en las tasas de lactancia materna en Brasil. Método: se trata de una revisión narrativa de la literatura desarrollada a través de la discusión de la literatura sobre el objetivo de esta reflexión. Resultados: se encontraron ocho estudios que respondieron a la pregunta orientadora del estudio, seis de los cuales evaluaron el impacto de la Rede Amamanta Brasil y dos de la Estrategia Alimentaria Brasileña de Lactancia Materna en las tasas de lactancia materna. Del total, seis fueron artículos, un informe de investigación y una disertación. Conclusión: los hallazgos fueron aparentemente contradictorios: si bien algunos estudios mostraron una mejora en las tasas de lactancia materna, otros no encontraron un aumento en la prevalencia en las unidades que se unieron a Rede Amamanta Brasil, ahora llamada Estrategia Alimentaria Brasileña de Lactancia Materna.

Palabras clave: Lactancia materna; Políticas de salud; Atención primaria de salud.

\section{Introdução}

O Aleitamento Materno (AM) é a estratégia isolada que tem o maior impacto na redução da mortalidade infantil (Brasil, 2015; Sankar et al., 2015). De acordo com a Organização Mundial da Saúde (OMS) e o Fundo das Nações Unidas para a Infância (UNICEF), milhões de crianças são salvas por ano graças ao Aleitamento Materno Exclusivo (AME) (United Nations Children's Fund, 2015).

Apesar dos avanços, as metas propostas pela OMS e Ministério da Saúde (MS) que preconizam a amamentação por dois anos ou mais, sendo de forma exclusiva nos primeiros seis meses de vida, ainda estão distantes de serem cumpridas (Guimarães et al., 2017; Brasil, 2015). O aumento nos indicadores de AM tem sido relacionado à implantação e implementação de políticas públicas direcionadas para o incentivo ao AM. A realização periódica de pesquisas sobre amamentação é um instrumento indispensável de avaliação de resultados de programas desenvolvidos, bem das intervenções mais adequadas a cada cenário (Brasil, 2017).

Desde década de 1980 as ações desenvolvidas no Brasil visando o estímulo do AM enfocavam a rede hospitalar. Por isso, em 2008, durante a abertura da Semana Mundial da Amamentação foi proposta a implementação da Rede Amamenta Brasil (RAB) direcionada à atenção básica. No mesmo ano foi assinada a Portaria MS/GM no 2.799 instituindo a RAB no âmbito do Sistema Único de Saúde (SUS) (Brasil, 2011). A RAB foi uma estratégia de promoção, proteção e apoio ao AM que previa que o cuidado também devia ser direcionado à Saúde da Mulher, pensando na família, já que os benefícios do AM atingem toda a sociedade (Brasil, 2011).

Em 2012, a integração da RAB com a Estratégia Nacional de Promoção da Alimentação Complementar Saudável (ENPACS) resultou na Estratégia Amamenta e Alimenta Brasil (EAAB). Com a iniciativa, o MS buscava estimular a promoção do aleitamento materno e da alimentação saudável para crianças menores de dois anos no âmbito do SUS (Brasil, 2015).

A EAAB foi formulada com base nas políticas e programas já existentes, como a Política Nacional de Atenção Básica (PNAB), a Política Nacional de Promoção da Saúde (PNPS), a Política Nacional de Alimentação e Nutrição (PNAN) e a Política Nacional de Aleitamento Materno (PNAM). A responsabilidade coube a Coordenação-Geral de Alimentação e Nutrição e a Coordenação de Saúde da Criança e Aleitamento Materno (COCAM/DAPES/SAPS) e como parceiros as secretarias estaduais e municipais de saúde (Brasil, 2015).

A Estratégia enfocou a qualificação do processo de trabalho dos profissionais da Atenção Primária a Saúde (APS), buscando a obtenção das metas referentes ao AM. Para isso, buscou capacitar a rede básica para acolher e oferecer assistência de qualidade para as mulheres, seus filhos e suas famílias. Tal estratégia tem sua metodologia estruturada a partir da problematização do processo de trabalho das Unidades Básicas de Saúde (UBS), valorizando a experiência de vida de todos os atores. Enfim, busca-se a transformação das práticas profissionais considerando as demandas em saúde da população diante das s inúmeras interfaces do AM que compõem a rede sociobiológica da amamentação (Brasil, 2017; 2015; 2011).

Para a implantação da EAAB, o processo se iniciou-se com a formação de facilitadores pelo Ministério da Saúde, os 
quais apoiam a formação de tutores das Secretarias Estaduais de Saúde (SES). Por sua vez, o tutor é um dos principais pilares de sustentação da Estratégia, com uma função primordial: realizar as oficinas de trabalho para implantação da Estratégia nas UBS, acompanhar o processo de implantação promovendo atividades que permitam a equipe pensar no seu processo de trabalho, além do monitoramento da implantação (Brasil, 2015; Venâncio et al., 2013).

O objetivo é a certificação das UBS que aderirem à EAAB. Para que isso ocorra, elas precisam cumprir alguns critérios: contar com a participação de pelo menos $85 \%$ da equipe nas oficinas; monitorar os índices de aleitamento materno e de alimentação complementar; dispor de instrumento de organização do cuidado à saúde da criança (protocolo ou fluxograma); cumprir a Norma Brasileira de Comercialização de Alimentos para Lactentes e Crianças de $1^{\text {a }}$ Infância, Bicos, Chupetas e Mamadeiras (NBCAL); desenvolver ações sistemáticas, individuais ou coletivas, para a promoção do aleitamento materno e da alimentação complementar saudável e cumprir pelo menos uma ação de incentivo ao aleitamento materno ou alimentação complementar saudável pactuadas no plano de ação, desde a primeira oficina. A certificação tem validade de um ano, ao término do qual a UBS deverá apresentar comprovação de que continua cumprindo os critérios (Brasil, 2015).

Nesse sentido, a EAAB vem de acordo com a proposta de educação permanente em saúde e a participação das UBS se dá com a realização de uma oficina de trabalho, durante a qual toda a equipe discute o seu processo de trabalho em relação às ações de promoção, proteção e apoio ao AM. Assim, cada equipe expõe suas dificuldades e acertos e busca as soluções a partir da sua realidade. O profissional de saúde que atua junto à mulher que amamenta deve ter habilidade científica, técnica e de relacionamento para assistir, além da mulher, o seu companheiro, filhos, família e comunidade (Brasil, 2015).

Por essa razão, o referencial teórico da EAAB é a educação crítico-reflexiva, reconhecendo o caráter histórico, social, político e econômico dos sujeitos. Busca-se estimular a participação e o empoderamento dos atores em busca de transformações sociais e privilegiar as experiências dos sujeitos envolvidos (Linhares et al., 2014).

Diante do exposto, o objetivo do presente estudo é refletir sobre o impacto da Rede Amamenta Brasil e da Estratégia Amamenta e Alimenta Brasil sobre os índices de aleitamento materno no Brasil.

\section{Metodologia}

Trata-se de uma revisão narrativa da literatura, a qual é utilizada para discutir e produzir síntese de publicações científicas prévias sobre certo tema, sendo indicada quando a questão de pesquisa não seja tão específica a ponto de ser abordada com o desenho de revisão sistemática (Green et al., 2006).

Realizou-se a busca das produções pelas pesquisadoras, tendo a seguinte pergunta de pesquisa: "Qual o impacto da Rede Amamenta Brasil e da Estratégia Amamenta e Alimenta Brasil sobre os índices de aleitamento materno no Brasil?”.

Os Descritores em Ciências da Saúde utilizados para a busca de documentos em formato eletrônico, sem delimitação de ano foram "Aleitamento Materno", "Atenção Primária à Saúde”, "Políticas Públicas de Saúde”, além das palavras-chave: "Rede Amamenta Brasil", "Estratégia Amamenta e Alimenta Brasil".

Assim, dividiu-se o estudo em etapas, a saber: pesquisa dos artigos nas bases de dados por meio dos descritores e palavras-chaves selecionados; leitura dos títulos e dos resumos para verificar a convergência do material à temática de estudo; leitura do artigo na íntegra; busca e leitura dos estudos originais encontrados por meio das referências finais dos artigos oriundos da busca nas bases de dados; leituras de manuais ou diretrizes oriundas da OMS e/ou do MS. Realizaram-se, após todas as leituras, a compilação dos materiais, seguida da análise e identificação dos temas para a reflexão e, finalmente, a elaboração das sínteses reflexivas do estudo.

Assim, esse estudo apresenta tem como eixo condutor a Rede Amamenta Brasil e a Estratégia Amamenta e Alimenta Brasil e a síntese aborda o seu impacto sobre o aleitamento materno. 


\section{Resultados}

Foram encontrados foram oito estudos que respondiam à questão norteadora do estudo, sendo seis que avaliavam a RAB e dois a EEAB.

Quadro 1 - Distribuição das publicações sobre o impacto da Rede Amamenta Brasil e da Estratégia Amamenta e Alimenta Brasil sobre os índices de aleitamento materno no Brasil.

\begin{tabular}{|c|c|c|c|}
\hline Publicação & Tipo & Objetivo & Resultados Principais/ Conclusão \\
\hline $\begin{array}{l}\text { 1. Brasil. Ministério da } r \text { Saúde. } \\
\text { Secretaria de Atenção à Saúde. } \\
\text { Departamento de Ações Programáticas } \\
\text { Estratégicas. (2013). Análise de } \\
\text { implantação da Rede Amamenta Brasil: } \\
\text { Relatório de pesquisa. Brasília: } \\
\text { Ministério da } \\
\text { https://bvsms.saude.gov.br/bvs/publicac } \\
\text { oes/analise_implantacao_amamenta_br } \\
\text { asil_relatorio.pdf }\end{array}$ & $\begin{array}{l}\text { Relatório de } \\
\text { pesquisa }\end{array}$ & $\begin{array}{l}\text { Avaliar o processo } \text { de } \\
\text { implantação } \text { da Rede } \\
\begin{array}{l}\text { Amamenta Brasil em três } \\
\text { municípios brasileiros. }\end{array}\end{array}$ & $\begin{array}{l}\text { Identificou-se baixa priorização da Rede nos } \\
\text { planos estaduais, as dificuldades no processo } \\
\text { de discussão com regionais de saúde e } \\
\text { municípios, a concorrência exercida por } \\
\text { outros projetos e programas de governo e a } \\
\text { ausência de recursos financeiros para } \\
\text { viabilizar a implantação da Rede Amamenta } \\
\text { Brasil. merece destaque o importante papel } \\
\text { que desempenha a coordenação técnica da } \\
\text { Rede no nível municipal. }\end{array}$ \\
\hline $\begin{array}{l}\text { 2. Passanha, A., Benício, M. H. D., } \\
\text { Venâncio, S. I., \& Reis, M. C. G. } \\
\text { (2013). Implantação da Rede } \\
\text { Amamenta Brasil e prevalência de } \\
\text { aleitamento materno exclusivo. Revista } \\
\text { de Saúde Pública, 47(6), 1141-1148. } \\
\text { https://doi.org/10.1590/S0034- } \\
8910.2013047004807\end{array}$ & Artigo & $\begin{array}{l}\text { Analisar a associação entre } \\
\text { grau de implantação da Rede } \\
\text { Amamenta Brasil e } \\
\text { prevalência de aleitamento } \\
\text { materno exclusivo. }\end{array}$ & $\begin{array}{l}\text { A prevalência de aleitamento materno } \\
\text { exclusivo em menores de } 6 \text { meses foi maior } \\
\text { nos locais certificados na Rede, evidenciando } \\
\text { a relevância de investir na certificação de } \\
\text { Unidades Básicas de Saúde nessa ação. }\end{array}$ \\
\hline $\begin{array}{l}\text { 3. Venâncio, S. I., Martins, M. C. N., } \\
\text { Sanches, M. T. C., Almeida, H., Rios, } \\
\text { G. S., \& Frias, P. G. (2013). Análise de } \\
\text { implantação da Rede Amamenta Brasil: } \\
\text { Desafios e perspectivas da promoção do } \\
\text { aleitamento materno na atenção básica. } \\
\text { Cadernos de Saúde Pública, 29(11), } \\
\text { 2261-2274. } \\
\text { https://doi.org/10.1590/0102- } \\
\text { 311x00156712 }\end{array}$ & Artigo & $\begin{array}{l}\text { Avaliar a implantação da } \\
\text { Rede Amamenta Brasil, } \\
\text { estratégia elaborada pelo } \\
\text { Ministério da Saúde para o } \\
\text { incentivo à amamentação na } \\
\text { atenção básica. }\end{array}$ & $\begin{array}{l}\text { Foi possível observar a influência do contexto } \\
\text { sobre a implantação da estratégia, de modo } \\
\text { que se evidenciou relação entre contextos } \\
\text { mais favoráveis e graus de implantação mais } \\
\text { avançados. }\end{array}$ \\
\hline $\begin{array}{l}\text { 4. Bastos, E. F. P., Souza, R. S. A., } \\
\text { Pereira, T. S. S., \& Molina, M. C. B. } \\
\text { (2014). Aleitamento materno e práticas } \\
\text { alimentares em crianças menores de um } \\
\text { ano em Vitória-ES. Revista Brasileira } \\
\text { de Pesquisa em Saúde, 16(2), 101-108. } \\
\text { https://doi.org/10.21722/rbps.v0i0.9292 }\end{array}$ & Artigo & $\begin{array}{l}\text { Analisar e comparar dados de } \\
\text { pesquisas sobre AM e práticas } \\
\text { alimentares de crianças } \\
\text { menores de um ano no } \\
\text { município de Vitória, Espírito } \\
\text { Santo, nos anos de } 2008 \text { e } \\
\text { 2012. }\end{array}$ & $\begin{array}{l}\text { Houve mudanças na prevalência de AME e } \\
\text { AM com o passar dos anos, demonstrando } \\
\text { que ações realizadas para o incentivo da } \\
\text { amamentação, principalmente as oficinas da } \\
\text { Rede Amamenta Brasil, são de extrema } \\
\text { importância. }\end{array}$ \\
\hline 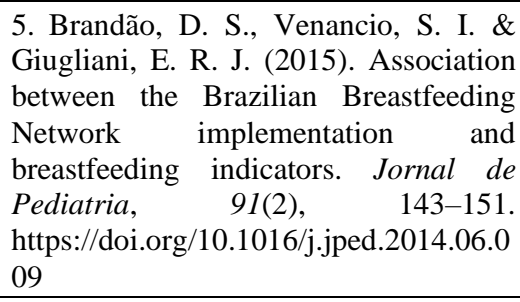 & Artigo & $\begin{array}{l}\text { Estimar a associação entre a } \\
\text { implementação da Rede } \\
\text { Amamenta Brasil e as } \\
\text { prevalências de aleitamento } \\
\text { materno (AM) em um } \\
\text { município de médio porte do } \\
\text { sul do Brasil. }\end{array}$ & $\begin{array}{l}\text { Contrariando a hipótese, não houve } \\
\text { associação significativa entre a } \\
\text { implementação da Rede Amamenta Brasil e } \\
\text { as prevalências de AM e AME no município } \\
\text { estudado. É possível que as dificuldades } \\
\text { encontradas na implementação da Rede nesse } \\
\text { município tenham influenciado esse } \\
\text { resultado. }\end{array}$ \\
\hline 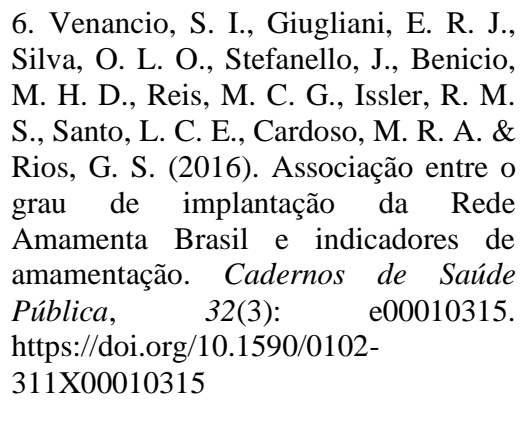 & Artigo & $\begin{array}{l}\text { Avaliar a implantação da } \\
\text { Rede Amamenta Brasil e seu } \\
\text { impacto sobre indicadores de } \\
\text { aleitamento materno (AM). } \\
\text { Realizou-se análise de } \\
\text { implantação de segundo tipo, } \\
\text { incluindo } 56 \text { unidades básicas } \\
\text { de saúde (UBS) de três } \\
\text { municípios brasileiros. }\end{array}$ & $\begin{array}{l}\text { Dificuldades para a implantação da Rede } \\
\text { Amamenta Brasil foram identificadas, e os } \\
\text { indicadores de AM variaram de acordo com o } \\
\text { número de critérios de certificação cumpridos } \\
\text { pelas UBS. As UBS cujo escore apontou } \\
\text { cumprimento dos quatro critérios de } \\
\text { certificação na RAB tiveram maior } \\
\text { prevalência de AME quando comparadas às } \\
\text { demais UBS. Entretanto, a maior prevalência } \\
\text { do AM em crianças de } 9-12 \text { meses ocorreu } \\
\text { entre as UBS que cumpriam dois critérios de } \\
\text { certificação. }\end{array}$ \\
\hline
\end{tabular}




\begin{tabular}{|c|c|c|c|}
\hline $\begin{array}{l}\text { 7. Tavares, J.S., Vieira, D.S., Dias, } \\
\text { T.K.C, Tacla, M.T.G.M, Collet, N., } \\
\text { Reichert, A.P.S. Logframe Model as } \\
\text { analytical tool for the Brazilian } \\
\text { Breastfeeding and Feeding Strategy. } \\
\text { Revista Nutrição. 2018;31(2):251-262. } \\
\text { http://dx.doi.org/10.1590/1678-98 } \\
\text { 652018000200010 }\end{array}$ & Artigo & $\begin{array}{l}\text { Analisar a implementação da } \\
\text { Estratégia Amamenta e } \\
\text { Alimenta Brasil em uma } \\
\text { capital do Nordeste brasileiro. }\end{array}$ & $\begin{array}{l}\text { Os principais problemas identificados na } \\
\text { EAAB foram as baixas taxas de aleitamento } \\
\text { materno exclusivo para crianças menores de } \\
\text { seis meses; curta duração da amamentação } \\
\text { total; introdução prematura de alimentos e } \\
\text { hábitos alimentares não saudáveis; e falta de } \\
\text { inserção de dados no SISVAN. As principais } \\
\text { causas desses problemas e os principais } \\
\text { fatores que comprometeram o } \\
\text { desenvolvimento das atividades planejadas } \\
\text { pelas equipes de saúde e, consequentemente, } \\
\text { a implantação da EAAB no município foram } \\
\text { a falta de apoio da gestão e a infraestrutura } \\
\text { inadequada das unidades de saúde. }\end{array}$ \\
\hline $\begin{array}{l}\text { 8. Bonini, t. P. L. (2019). Avaliação do } \\
\text { grau de implantação e os efeitos } \\
\text { observados na estratégia amamenta } \\
\text { alimenta brasil nas unidades de saúde } \\
\text { da família de Piracicaba/SP. } \\
\text { Dissertação (mestrado). Universidade } \\
\text { Estadual de Campinas. } \\
\text { Http://repositorio.unicamp.br/bitstream/ } \\
\text { reposip/335903/1/bonini_tatianadoprad } \\
\text { olima_m.pdf }\end{array}$ & Dissertação & $\begin{array}{l}\text { Avaliar a implantação da } \\
\text { EAAB nas unidades de saúde } \\
\text { da família, a partir do } \\
\text { monitoramento das ações } \\
\text { propostas pelas unidades e os } \\
\text { efeitos destas ações nos } \\
\text { indicadores de amamentação } \\
\text { e alimentação saudável. }\end{array}$ & $\begin{array}{l}\text { As ações propostas durante as oficinas de } \\
\text { implantação da EAAB foram efetivas para a } \\
\text { promoção e manutenção do AM, uma vez que } \\
\text { apresentaram impacto nos indicadores de } \\
\text { ame, ao compararmos com as unidades sem a } \\
\text { estratégia. Além disso, os resultados } \\
\text { demonstraram uma boa adesão das unidades } \\
\text { nas oficinas de implantação, com a } \\
\text { participação de } 80-85 \% \text { dos profissionais. }\end{array}$ \\
\hline
\end{tabular}

Fonte: Autores (2021).

\section{Discussão}

Sobre o impacto da RAB e da EAAB nas prevalências do AM, as pesquisas são aparentemente contraditórias. Em Ribeirão Preto (São Paulo), foi realizado o primeiro estudo para avaliar a associação entre o grau de implantação da RAB e a prevalência da AME, tanto nas unidades certificadas quanto nas em certificação e não certificadas pela RAB. Os resultados desse estudo apontaram que a prevalência de AME foi significativamente maior nas crianças acompanhadas em unidades de saúde certificadas na Rede, evidenciando a relevância de investir na certificação de UBS nessa ação (Passanha et al., 2013).

Outra investigação conduzida nos municípios de Dourados (Mato Grosso do Sul), Porto Alegre (Rio Grande do Sul) e Ribeirão Preto (São Paulo) também identificou que as UBS certificadas apresentavam melhor desempenho nas ações previstas pela RAB em comparação às UBS que haviam realizado a oficina de trabalho, sem, no entanto, terem sido certificadas até o momento das entrevistas. Em contrapartida, observou-se também que algumas UBS apesar de certificadas, deixaram de atender esses critérios segundo o relato dos gerentes (Venancio et al., 2016).

Investigação realizada em Porto Alegre (Rio Grande do Sul), Corumbá (Mato Grosso do Sul) e Brasília (Distrito Federal) avaliou a implantação da RAB e evidenciou que a promoção do AM não está plenamente implantada no âmbito da atenção básica. Apesar de a RAB ter exercido um impacto positivo nos indicadores de AM, era comum as UBS que já tinham iniciado o processo de certificação na Rede interrompê-lo ou apresentar baixo grau de execução dos critérios após alguns meses. Para os autores do estudo, se as ações de AM, no contexto de uma política nacional, fossem pactuadas entre as três esferas de governo, minimizaria dificuldades como a disputa por recursos e prioridade com outros projetos e programas nos estados e municípios (Venâncio et al., 2013).

Por outro lado, estudo de impacto da RAB em Bento Gonçalves (Rio Grande do Sul), avaliando a associação entre a implementação da RAB e as prevalências de AM em menores de um ano e de AME em menores de seis meses, não encontrou diferença estatisticamente significativa nas prevalências de AME e de AM entre as crianças acompanhadas por serviços que aderiram à RAB (certificados e não certificados) e as que frequentavam serviços que não foram expostos às ações da estratégia (Brandão et al., 2015).

Uma pesquisa solicitada pelo MS com o objetivo de identificar fatores facilitadores e restritivos da implantação da 
RAB apresentou recomendações quanto aos critérios de certificação das UBS nos níveis federal, estadual e municipal, tendo em vista o grande potencial da RAB para a mudança nos processos de trabalho das equipes da Atenção Básica e qualificação da atenção à dupla mãe-filho em AM (Brasil, 2013). Entretanto, apesar das recomendações oficiais sobre a certificação das unidades a literatura aponta que nem sempre há um impacto direito do processo na melhoria dos indicadores de AME e AM (Brandão et al., 2015).

Ainda assim, é importante que o processo seja sempre incentivado. O estudo realizado em Vitória (Espírito Santo) demonstrou que as ações de incentivo à amamentação devem ser contínuas, já que, com o passar dos anos, houve mudanças na prevalência de AME e AM. Apesar do aumento da prevalência, os resultados encontrados foram muito aquém das recomendações oficiais (Bastos et al., 2014).

No estudo realizado em Piracicaba (São Paulo), a fim de avaliar a implantação da EAAB, a partir do monitoramento das ações propostas pelas unidades e os efeitos destas ações nos indicadores de amamentação identificou-se que a maioria das equipes conseguiu cumprir e implementar total ou parcialmente as ações nas consultas médicas, de enfermagem e odontológicas, nos grupos de educação em saúde e visitas domiciliares, impactando positivamente na continuidade do AME (Bonini, 2019).

Na pesquisa realizada em Recife (Pernambuco) a partir da representação gráfica da árvore de problema da EAAB foi construído o diagrama com a estruturação da Estratégia que mostrou as fragilidades nas informações dos documentos oficiais da Estratégia, bem como problemas na sua implementação (Tavares et al, 2018).

As dificuldades encontradas na implementação da EEAB são semelhantes às da RAB devem ser analisadas no contexto do sistema de saúde brasileiro: a não manutenção do cumprimento dos critérios de certificação da RAB das unidades já certificadas, baixo grau de cumprimento dos critérios pela maioria das unidades que iniciaram o processo de certificação na Rede, mas que ainda não haviam sido certificadas; dificuldade relacionados aos recursos humanos e/ou materiais; sobrecarga e rotatividade dos profissionais de saúde; interrupção de programas e/ou mudanças relacionados a cada gestão, o que resulta, muitas vezes, na desmotivação do profissional de saúde (Brandão et al., 2015).

Essa (des)motivação, bem como o comprometimento do profissional de saúde estão diretamente relacionados aos resultados nos indicadores (Almeida et al., 2015). Também é importante que as relações com os profissionais de saúde sejam adequadas, pois quando as dificuldades com a amamentação surgem, especialmente em domicílio, as mulheres utilizam-nas para a tomada de decisão (Abreu et al., 2015). Considerando que a maioria das dificuldades com o AM ocorre no domicílio, reconhece-se o papel fundamental da equipe da Estratégia Saúde da Família (ESF), em destaque o enfermeiro, para assistir neste momento tão importante e pleno de transformações para a mãe, seu filho e toda a família.

A capacitação também é fundamental nesse processo já que dela depende o aumento dos escores de conhecimento e manejo do AM (Vasquez et al., 2015). A educação permanente em saúde é efetiva para melhorar o desempenho dos trabalhadores de saúde, que propiciam mudanças no processo de trabalho, refletindo na qualidade do aconselhamento materno (Relvas et al., 2019).

Desse modo, o investimento em ações estratégicas, que produzam transformações em favor do AM deve ser considerado como prioritário pelas políticas públicas de saúde (Bastos et al., 2014).

No ano de 2018, uma oficina da esfera federal identificou que com relação à certificação das UBS, foi sugerido que alguns critérios fossem revisados e que os sistemas de monitoramento fossem atualizados para otimizar os registros, bem como o processo de avaliação dos pedidos de certificação fosse descentralizado para os estados (Brasil, 2018). Nesse contexto, outra ação necessária é a sensibilização dos gestores municipais e estaduais para priorizar a implementação das ações, bem como planejamento para implementação, avaliação e monitoramento da EAAB (Bortolini, 2017).

Os estudos informam aspectos da implementação da EAAB que precisam de melhoria e refletem como é desafiador 
implementar uma estratégia em escala nacional de forma efetiva e sustentável a longo prazo (Melo, 2020).

Destaca-se que, desde a sua criação, não houve nenhum processo oficial de avaliação do impacto das ações desenvolvidas na EAAB e da sua influência na melhora da prevalência de AM (Melo, 2020).

Finalmente, a avaliação do impacto das ações da EAAB sobre a prevalência dos indicadores de AM constitui-se uma premissa para justificar a continuidade da sua implementação com base nos resultados de impacto apresentados.

\section{Conclusão}

Os achados foram aparentemente contraditórios: enquanto algumas pesquisas demostraram prevalências de AM maior nas crianças acompanhadas em unidades de saúde certificadas, outros estudos não encontraram diferenças entre as crianças acompanhadas por serviços que aderiram à $\mathrm{RAB}$ ou a EAAB (certificados e não certificados) e as que frequentavam serviços que não foram expostos às ações da estratégia.

Espera-se com esse estudo disseminar a importância a importância da avaliação e do monitoramento das estratégias de incentivo ao aleitamento materno no impacto dos indicadores. Recomenda-se a realização de outros estudos de avaliação do impacto da RAB e da EAAB, que explorem seu efeito, controlando a influência de outras variáveis individuais e contextuais.

A limitação deste estudo refere-se à escassez de publicações relacionadas ao impacto da RAB e da EAAB sobre os índices de aleitamento materno, apesar da grande quantidade de estudos sobre os benefícios da amamentação. O tema carece, ainda, de futuras investigações para avaliação da sua eficácia na prática profissional.

\section{Referências}

Abreu, F. C. P., Marski, B. S. L., Custódio, N., Carvalho, S. C., \& Wernet, M. (2015). Breastfeeding preterm infants at home. Texto \& Contexto Enfermagem, 24(4), 968-975. https://doi.org/10.1590/0104-0707201500000300014

Almeida, J. M., Luz, S. A. B., \& Ued, F. V. (2015). Apoio ao aleitamento materno pelos profissionais de saúde: Revisão integrativa da literatura. Revista Paulista de Pediatria, 33(3), 355-362. https://doi.org/10.1016/j.rpped.2014.10.002

Bastos, E. F. P., Souza, R. S. A., Pereira, T. S. S., \& Molina, M. C. B. (2014). Aleitamento materno e práticas alimentares em crianças menores de um ano em Vitória-ES. Revista Brasileira de Pesquisa em Saúde, 16(2):101-108. https://doi.org/10.21722/rbps.v0i0.9292

Bonini, T. P. L. (2019). Avaliação do grau de implantação e os efeitos observados na Estratégia Amamenta Alimenta Brasil nas Unidades de Saúde da Família de Piracicaba/SP. Dissertação Campinas. http://repositorio.unicamp.br/bitstream/REPOSIP/335903/1/Bonini_TatianaDoPradoLima_M.pdf

Bortolini, G. A. (2017). Avaliação da implementação da Estratégia Amamenta e Alimenta Brasil (EAAB). Trabalho de conclusão de curso (Especialização em Gestão Pública na Saúde), Universidade de Brasília. https://bdm.unb.br/handle/10483/17578

Brandão, D. S., Venancio, S. I. \& Giugliani, E. R. J. (2015). Association between the Brazilian Breastfeeding Network implementation and breastfeeding indicators. Jornal de Pediatria, 91(2), 143-151. https://doi.org/10.1016/j.jped.2014.06.009

Brasil. Ministério da Saúde. Secretaria de Atenção à Saúde. (2015). Estratégia Nacional para Promoção do Aleitamento Materno e Alimentação Complementar Saudável no Sistema Único de Saúde: Manual de implementação. Brasília: Ministério da Saúde. https://bvsms.saude.gov.br/bvs/publicacoes/estrategia_nacional_promocao_aleitamento_materno.pdf

Brasil. Ministério da Saúde. Secretaria de Atenção à Saúde. Área Técnica de Saúde da Criança e Aleitamento Materno. (2011). Rede Amamenta Brasil: Os primeiros passos (2007-2010). Brasília: Ministério da Saúde. https://bvsms.saude.gov.br/bvs/publicacoes/rede_amamenta_brasil_primeiros_passos.pdf

Brasil. Ministério da Saúde. Secretaria de Atenção à Saúde. Departamento de Ações Programáticas Estratégicas. (2013). Análise de implantação da Rede Amamenta Brasil: Relatório de pesquisa. Brasília: Ministério da https://bvsms.saude.gov.br/bvs/publicacoes/analise_implantacao_amamenta_brasil_relatorio.pdf

Brasil. Ministério da Saúde. Secretaria de Atenção à Saúde. Departamento de Ações Programáticas Estratégicas. (2017). Bases para a discussão da política nacional de promoção, proteção e apoio ao aleitamento materno. Brasília: Ministério da Saúde. https://bvsms.saude.gov.br/bvs/publicacoes/bases_discussao_politica_aleitamento_materno.pdf

Brasil. Ministério da Saúde. Coordenação Geral de Alimentação e Nutrição/CGAN e Coordenação Geral de Saúde da Criança e Aleitamento Materno/CGSCAM (2018). Oficina de Escuta da EAAB: Relatório. Brasília: Ministério da Saúde.

Green, B. N., Johnson, C. D., \& Adams, A. (2006). Writing narrative literature reviews for peer-reviewed journals: Secrets of the trade. Journal of Chiropractic Medicine, 5(3), 101-117. https://doi.org/10.1016/S0899-3467(07)60142-6 
Research, Society and Development, v. 10, n. 10, e339101018941, 2021

(CC BY 4.0) | ISSN 2525-3409 | DOI: http://dx.doi.org/10.33448/rsd-v10i10.18941

Guimarães, C. M. S., Conde, R. G., Brito, B. C., Gomes-Sponholz, F. A., Oriá, M. O. B., \& Monteiro, J. C. S. (2017). Comparação da autoeficácia na amamentação entre puérperas adolescentes e adultas em uma maternidade de Ribeirão Preto, Brasil. Texto \& Contexto - Enfermagem, 26(1). https://doi.org/10.1590/0104-07072017004100015

Linhares, F. M. P., Pontes, C. M., \& Osório, M. M. (2014). Construtos teóricos de Paulo Freire norteando as estratégias de promoção à amamentação. Revista Brasileira de Saúde Materno Infantil, 14, 433-439. https://doi.org/10.1590/S1519-38292014000400013

Melo, D. S. (2020). Processo de implementação da Estratégia Amamenta e Alimenta Brasil: Uma análise do caminho de impacto do programa [Mestrado em Nutrição em Saúde Pública, Universidade de São Paulo]. https://doi.org/10.11606/D.6.2020.tde-02022021-170552

Passanha, A., Benício, M. H. D., Venâncio, S. I., \& Reis, M. C. G. (2013). Implantação da Rede Amamenta Brasil e prevalência de aleitamento materno exclusivo. Revista de Saúde Pública, 47(6), 1141-1148. https://doi.org/10.1590/S0034-8910.2013047004807

Relvas, G. R. B., Buccini, G. S., \& Venancio, S. I. (2019). Avaliação do uso de um manual de apoio à implementação da "Estratégia Amamenta e Alimenta Brasil"', DEMETRA: Alimentação, Nutrição \& Saúde, 14(0), 43322. https://doi.org/10.12957/demetra.2019.43322

Sankar, M. J., Sinha, B., Chowdhury, R., Bhandari, N., Taneja, S., Martines, J., \& Bahl, R. (2015). Optimal breastfeeding practices and infant and child mortality: A systematic review and meta-analysis. Acta Paediatrica (Oslo, Norway: 1992), 104(467), 3-13. https://doi.org/10.1111/apa.13147

Tavares, J. S., Vieira, D. de S., Dias, T. K. C., Tacla, M. T. G. M., Collet, N., \& Reichert, A. P. S. (2018). Logframe Model as analytical tool for the Brazilian Breastfeeding and Feeding Strategy. Revista de Nutrição, 31, 251-262. https://doi.org/10.1590/1678-98652018000200010

United Nations Children's Fund. (2015). Breastfeeding Advocacy Initiative: For the best start in life. UNICEF. https://www.unicef.org/nutrition/files/Breastfeeding_Advocacy_Strategy-2015.pdf

Vasquez, J., Dumith, S. C., \& Susin, L. R. O. (2015). Aleitamento materno: Estudo comparativo sobre o conhecimento e o manejo dos profissionais da Estratégia Saúde da Família e do Modelo Tradicional. Revista Brasileira de Saúde Materno Infantil, 15(2), 181-192. https://doi.org/10.1590/S151938292015000200004

Venancio, S. I., Giugliani, E. R. J., Silva, O. L. O., Stefanello, J., Benicio, M. H. D., Reis, M. C. G., Issler, R. M. S., Santo, L. C. E., Cardoso, M. R. A. \& Rios, G. S. (2016). Associação entre o grau de implantação da Rede Amamenta Brasil e indicadores de amamentação. Cadernos de Saúde Pública, 32(3): e00010315 https://doi.org/10.1590/0102-311X00010315

Venâncio, S. I., Martins, M. C. N., Sanches, M. T. C., Almeida, H., Rios, G. S., \& Frias, P. G. (2013). Análise de implantação da Rede Amamenta Brasil: Desafios e perspectivas da promoção do aleitamento materno na atenção básica. Cadernos de Saúde Pública, 29(11), 2261-2274. https://doi.org/10.1590/0102-311x00156712 